\title{
Exploration on the College Mathematics Foundational Curriculum Reform by Using Mathematical Software
}

\author{
He Shan, Shao Lu and Tan Hongxin \\ Fisheries and Life Science College, Shanghai Ocean University, Shanghai 201306, China \\ s_he@shou.edu.cn, hxtan@shou.edu.cn
}

\begin{abstract}
As professional basic courses for specialties in fields of science and engineering, the mathematics foundational courses are always unsatisfactory in instruction effect and learning experience. This paper narrates the mode and significance of software-based mathematics foundational courses, and elaborates four essential footholds of measuring students' knowledge acquaintance, integrating disciplinary background, meeting the needs of extracurricular activities and the needs of work and study in future. In the end we put forward four promotion recommendations for the software-based mathematics foundational courses that are strengthening disciplinary support, implementing stratified teaching, updating the credit authentication and reinforcing the resource coordination.
\end{abstract}

Index Terms - Mathematics foundational courses, Mathematical software, Disciplinary support, Curriculum reform

\section{Introduction}

The mathematics foundation courses are the fundamental courses for professions of science, engineering, agriculture and medicine in domestic colleges which lays the foundation for students mastering knowledge of other subjects. In the course of mathematics foundational course instruction exist serious problems such as teachers' time over-investing, students' receiving difficulty and teaching management dilemma. Therefore, what kind of effective measures can be taken into teaching quality promotion of mathematics foundational courses can't be avoided for most domestic colleges. For the recent years, some vocational colleges try to teach the mathematics foundational course combining mathematical software and it is effective. The practice of Shandong Industrial Vocational College proved that it has far better effect to combine mathematical software of MathType than complete traditional instruction and students of graphic printing professional can roughly master the knowledge of data edition within 45 minutes [1]. Other scholars proposed to assist blackboard writing and opening mathematical experiments by using mathematical software [2]. Therefore, mathematics foundational curriculum instruction with mathematical software can be regarded as a solution to this problem which can lead to a win-win situation of the teacher, the student and the college. Given this, this paper has stated the mode and significance and four footholds of carrying our software-based mathematics foundational course instruction and discussed measures and suggestions in order to provide a scientific reference to the instruction innovation of mathematics foundational courses in colleges.

\section{The Teaching Status of Mathematics Foundational Courses in Colleges}

College mathematics foundational courses include quite varieties such as Advanced Mathematics, Linear Algebra, Probability Theory\& Mathematical Statistics and so on. There are several problems including teachers' superabundant input, students' accepting obstacle and management dilemma of administration. From the perspective of teaching, teachers always contribute much time on homework correcting, afterschool Q\&A, test paper producing, examination arrangement and paper checking of mid-term and final examinations except normal class time. Take the mathematics foundational courses of Shanghai Ocean University as example, most teachers assign homework every week and correct and comment homework in class and then make students to revise them after class. And specialized Q\&A in night class is opened regularly. Although so much has been focused on this work, the performance of aquatic professions remains poor which can be proved by the fact that the average scores of Advanced Mathematics, Linear Algebra, Probability Theory\& Mathematical Statistics are about 75 for the recent 4 years among which the average score of Linear Algebra is just above that of the other two but is still lower than 80. From the student's learning experience, the mathematics foundational courses have high challenge, severe subnormal input- output ratio and poor application prospect of knowledge with which students consider negatively and tackle difficult knowledge points in a eluding manner and that why students feel insufficient of capacity in mathematics teaching practice. In Shanghai Ocean University, we arranged the course of Advanced Mathematics into the first and second semester for 4 credits each and then a question appeared that the failure rate in second semester is 10 percent higher than that in first semester no matter counted by grades or professions which can be generated by three factors.

\subsection{The supporting role of former knowledge base is gradually getting weaker}

When students are learning mathematics foundational courses in the first semester of college, it's easy for them to utilize mathematical knowledge acquired in high school such as calculus, permutation and combination. Therefore even though they don't endeavor to study they can still pass the exams just rely on the old knowledge in high school period. Until this time these students have already accumulated much study obstacles which hinder them from further learning so as they are easy to fall into a vicious spiral. 


\subsection{Students lack self-control and time arrangement ability}

The college mathematics foundational courses are generally set in the first academic year, but some students feel hard to adapt to the mode of selecting courses and study all by their own from the high school to the university. On one hand, the class timetable of second semester is always overfilled because of students' lack of experience in course selecting which will cause the dispersion of mathematics learning time and study focus therefore it's hard for them to concentrate to study mathematics. On the other hand, the number of associations and extracurricular activities is too large to make some students unable to balance study and extracurricular activities which will cause them to regret just when test results come out.

\subsection{The necessity recognition of knowledge and learning process is too low}

Nowadays undergraduates can acquire intensive and massive knowledge via internet due to the highly-developed global informatization in which they developed the habit to filter critical information unknowingly and pay much attention on the function and effectiveness of information. From teachers' perspective, it's difficult to persuade students of knowledge's usefulness because of the fundamental property of mathematics which means it has a distance to reality application.

\section{Application Suggestion of Mathematical Software in the College Mathematics Foundational Courses}

To carry out software-based mathematics foundational courses is mainly to introduce the embodiment of knowledge points in software when imparting theoretical knowledge and to introduce theoretical knowledge contained in software while explaining software functions. Take the course of Probability Theory\& Mathematical Statistics as example, when teaching the point of Analysis of Variance, it's suggested to open the one-way AVONA function of SPSS and explain how the SPSS analysis process embodies the knowledge point and how to use the software to complete variance analysis. In this way, students can deepen the image of knowledge point by demonstration using mathematical software; they can understand the application range and operational process of knowledge points easily and have more recognition about knowledge usefulness by contrasting software and theoretical knowledge; they can reach an integration between theoretical and practical knowledge in class and grasp relative statistic skills while understanding knowledge.

\section{The Footholds to carry out Software-based Mathematics Foundational Courses}

\subsection{Mathematical software operation is one of standards to measure mathematics knowledge acquirement}

In knowledge area, theoretical and practical knowledge are built upon and supplement each other, in other words the theoretical comes from and also is applied to the practical. As the old saying goes, "The armchair strategist is unreliable and we should do it ourselves". So is mathematics, the ultimate aim and the embodiment of grasping mathematics is to skillfully use mathematical software and to solve realistic problems by software which is the primary starting point of utilizing software in mathematic classes.

\subsection{The software-based mathematics foundational instruction is beneficial to discipline integration}

To combine some examples of knowledge application in discipline and profession research while teaching mathematics foundational points can effectively upgrade the educational quality of mathematics foundational courses. Yet how to arrive to this prospection or the way to acquire examples is worth discussing. College mathematics foundational courses are generally opened by Information Institute or Mathematics and Physics Institute for relative professions of the whole college while teachers of mathematics foundational courses and teachers of professional courses need more communication. Mathematics teachers hope professional teachers to provide some instructional practical cases in some colleges. Indeed, case-combined instruction will be more interesting and lively, but it's not practicable to accumulate cases by this way. Firstly, the subject of collecting cases is wrong. Professional teachers know little about mathematics knowledge points and there are few occasion for them to use single mathematics point in discipline or profession research. Secondly, the way to collect cases is wrong. If we set the professional teacher number as $\mathrm{n}$ and mathematics point number as $\mathrm{m}$, even though every teacher feedbacks 1 case for every point, then the total feedback frequency will reach to more than $\mathrm{n}^{*} \mathrm{~m}$. What's more, mathematics teachers will take nearly the same quantity of work in information receiving which will lead to an exceptional enormous labor amount and go against disciplinary case collection. Because mathematical software is widespread used in scientific research and the final form of research is always thesis or treatise, it's suggested to collect practical cases of software application in professional teachers' research achievement by mathematics teachers which should be the best way to acquire information and largely reduce repetitive work amount. And using mathematical software in class will organically connect theoretical points with professional cases.

\subsection{Learning mathematical software meets the need of extra- curricular outreach activities}

The rapid development of economical society accelerated the diversification and inter-disciplinarity process of talent cultivation for which many colleges vigorously encourage students to participate in the Undergraduate Innovation Program, all types of discipline competitions and advise them to enter mentors' experiment labs to launch scientific research as soon as possible. When students participate most activities mentioned above, practical mathematics knowledge such as Mathematical Statistic is involved among which the demand for mastering mathematical software is pretty critical in some mathematical competition such as the Mathematical Contest in Modeling. The majority of participants were sophomores in the past while more and more freshmen take part in these activities for recent years. Therefore, the student's need for 
mastering mathematical software becomes earlier and more urgent. By contrast, the opening time of practical mathematics courses is commonly at the second academic year without combining applying mathematical software which makes students carrying out scientific research, innovation activities and competitions in the first two academic years face the shortage of instrumental knowledge. The software-based mathematics foundational courses will mitigate this condition for the most part.

\subsection{Mastering mathematical software is beneficial to the work and study in the future \\ Mathematics is the basement of all scientific research, so} it's everywhere in daily study, research and work and some practical mathematical tools are necessary in different walks of life. For example, the SPSS based on the Probability Theory\& Mathematical Statistics is widely used in data summarization and analysis in all walks of life and it's almost indispensable in data processing of kinds of questionnaires. If we teach the Probability Theory \& Mathematical Statistics combining applying SPSS, it will radically resolve the problem and to promote the interflow and integration of theoretical knowledge memory and manipulating statistic software. That is to say, it both deepen the image of theoretical points and facilitate proficiency of operational skill.

\section{The Measures and Suggestions in carrying out Software-based Mathematics Foundational Courses}

\subsection{Collecting teaching materials in the discipline area}

Both support of disciplinary cases and applying mathematical software are crucial factors to elevate teaching effectiveness of mathematics foundational courses and the two complement each other well. The effectiveness of applying mathematical software depends on the elaboration, vividness, practicality and the knowledge point integrating degree of disciplinary supporting data. While collecting disciplinary and professional cases in practice relies on mathematics teachers' analysis on professional teachers' research achievements and their extraction of proper cases supporting or corroborating some mathematics points which will enrich vivid case bank for software-based mathematics foundational courses and increase students' enthusiasm and professional master.

\subsection{Adopting stratified teaching and personalized teaching}

Stratified teaching is an effective approach to individualized order-type cultivation. Take Shanghai Ocean University as example, we prepare a mathematics diagnostic test for Advanced Mathematics A at the beginning of the entrance, and then distribute these students into three classes of $\mathrm{A}, \mathrm{B}$ and $\mathrm{C}$ according to the principle of stratified teaching. This mode will facilitate teachers' better preparation for students' situation. When setting up teaching scaffold, teachers can establish the teaching content difficulty easier and there's less portion of students feeling hungry or overfull to learn in general teaching mode because of students' similar learning foundation. Adopting stratified teaching undoubtedly lays a solider platform for applying mathematical software in mathematics foundational courses. It's suggested to expand the stratified teaching mode tried on Advanced Mathematics A to other mathematics foundational courses so as to further elevate the relevancy of students' learning demand and teaching content and to improve class effectiveness.

\subsection{To innovate and reform the credit authentication mode}

In most colleges inland nowadays, the class learning atmosphere remains to be strengthened. While the key point of improving learning atmosphere relies on turning asked to learn to need to learn which will fulfill students' learning consciousness. The high failure rate of mathematics foundational courses makes a part of students increase fear even psychological barriers further more. Thus, if students can get corresponding credits of mathematical software courses by getting passed in software-based mathematics foundational courses, they will be greatly encouraged of learning initiative and positivity and more cherish and care the chance to take part in the mathematics foundational class.

\subsection{Integrating teaching resources effectively by comprehensive arrangement}

Mathematical courses have large numbers and different names and belong to various curriculum modules in many colleges. However some mathematical courses enjoy similar or overlapping content which will cause some problems. On one hand, the course resources scattered and students have to choose quite number of homogeneous courses to reach the knowledge integrity which is also a kind of resource wasting from the perspective of resource allocation. On the other hand, some students are opportunistic and they choose mathematical courses enjoying basically same or similar content which belong to different curriculum modules to acquire multiple credits, and this is definitely against administrators' original willing. In Shanghai Ocean University, mathematical courses include Advanced Mathematics, Linear Algebra, Probability Theory\& Mathematical Statistics in disciplinary compulsory module, Biostatistics in disciplinary elective module, mathematical software Mathematica and Matlab in comprehensive elective module. If these resources can be sorted and integrated, it's positive for the college, teachers and students and will promote further application of software in mathematics foundational classes.

Undeniably, the variety of teaching methods and teaching patterns has already greatly enriched the mathematics foundational classes, and the informatization not only penetrates into every corner of social life but also become a fresh color for perfecting teaching methods. The informatization of mathematics foundational courses by applying mathematical software will lay a firmer foundation in teaching effective elevating and learning experience optimizing.

\section{Acknowledgements}

This work, in correspondence with Tan Hong-Xin, is contributed equally by Shao Lu and financially supported by Program of Shanghai Municipal Education Commission (No. B-5008-13-0003) and 085 project (No. B-5008-12-0101-4). 


\section{References}

[1] Y. Shi, "Teaching research on informational mathematics courses combining software in vocational colleges," Science \& Technology Information, no. 14, pp. 128-129, July 2012.
[2] S. Gao, "On the application and effectiveness of mathematical software in mathematics courses in the vocational college," Education Exploration, no. 8, pp. 35-37, August 2009. 